\title{
SGLT2 inhibitors and protection against pancreatic beta cell failure
}

\author{
Shun-ichiro Asahara ${ }^{1}\left[\right.$. Wataru Ogawa ${ }^{1}$
}

Received: 10 August 2018 / Published online: 17 September 2018

(C) The Japan Diabetes Society 2018

Type 2 diabetes is characterized by insulin resistance in peripheral tissues and pancreatic beta cell failure, with the inability of pancreatic beta cells to compensate for increasing insulin resistance resulting in the onset of diabetes. Pancreatic beta cell failure can be categorized as a qualitative or quantitative abnormality, which corresponds to a defect in the ability to secrete insulin and a reduction in pancreatic beta cell mass, respectively. Given that pancreatic beta cell mass has been found to be reduced even before the onset of type 2 diabetes [1], the contribution of this quantitative abnormality to the pathogenesis of type 2 diabetes has received much attention. Although a compensatory increase in pancreatic beta cell mass in response to systemic insulin resistance has been observed in obese Caucasian individuals [2], no such effect has been detected in obese Japanese [3], suggestive of a reduced capacity to increase beta cell mass in the Japanese population. Interventions that increase beta cell mass might thus be expected to contribute to the prevention or treatment of type 2 diabetes in Japanese individuals.

Some antidiabetic drugs exert pleiotropic effects independent of their glucose-lowering activity, with one such prominent action being the protective effect of sodium glucose cotransporter 2 (SGLT2) inhibitors on multiple organs. The EMPA-REG OUTCOME (Empagliflozin Cardiovascular Outcome Event Trial in T2DM Patients) and CANVAS (Canagliflozin Cardiovascular Assessment Study) trials have shown that SGLT2 inhibitors prevent the development of cardiovascular events as well as the progression of renal dysfunction in patients with type 2 diabetes $[4,5]$. These results, together with various other clinical and preclinical observations, have thus suggested that SGLT2 inhibitors protect against renal and cardiac damage. Moreover, this class

Shun-ichiro Asahara

asahara@med.kobe-u.ac.jp

1 Division of Diabetes and Endocrinology, Department of Internal Medicine, Kobe University Graduate School of Medicine, 7-5-1 Kusunoki-cho, Chuo-ku, Kobe 650-0017, Japan of drug appears to exert beneficial effects on other organs including beta cells of the pancreas.

Several studies have shown that SGLT2 inhibitors improve insulin secretion in patients with type 2 diabetes. The ratio of the incremental area under the plasma C-peptide concentration-versus-time curve to that under the plasma glucose concentration-versus-time curve during a 75-g oral glucose tolerance test was increased significantly in type 2 diabetic patients treated with dapagliflozin compared with those receiving a placebo [6]. The administration of empagliflozin to patients with type 2 diabetes was also shown to improve pancreatic beta cell function, as assessed by the insulin secretion/insulin resistance index during a hyperglycemic clamp [7]. Furthermore, the improvement in insulin secretion induced by ipragliflozin in Japanese patients with type 2 diabetes persisted even after the cessation of drug administration [8]. The mechanism underlying this beneficial effect of SGLT2 inhibitors on insulin secretion has. however, remained unknown.

Zucker diabetic fatty (ZDF) rats develop diabetes as a result of an age-dependent decline in beta cell mass associated with deterioration of pancreatic islet architecture. The administration of empagliflozin to these rats was found to ameliorate glucose intolerance and to attenuate the natural decline of insulin secretion [9]. In addition, it maintained the normal structure of pancreatic islets, whereas treatment with a glucagon-like peptide-1 (GLP-1) analog or sulfonylureas did not. In another animal model, the $\mathrm{db} / \mathrm{db}$ mouse, in which the number of pancreatic beta cells also declines with age, treatment with luseogliflozin increased beta cell proliferation and attenuated beta cell apoptosis [10]. These effects may be related to an associated increase in the expression of MafA and Pdx 1 , both of which are key transcription factors in the development and functional maintenance of beta cells. In NOD mice, a model of autoimmune-mediated type 1 diabetes, treatment with empagliflozin resulted not only in an improvement in blood glucose levels, but also in an increase in pancreatic beta cell mass, an effect that appeared to be related to attenuation of the accumulation of reactive oxygen species in beta cells [11]. Favorable effects of SGLT2 
inhibitors on pancreatic beta cells appear not to be limited to an increase in cell mass. Administration of dapagliflozin to individuals with type 2 diabetes was thus found to augment the secretion of insulin induced by exogenous GLP-1 or gastric inhibitory polypeptide (GIP) [12]. In addition, Xu et al. [13] reported that phlorizin treatment of pancreatectomized hyperglycemic rats recovered expression of GLP-1 receptor and GIP receptor, which was down-regulated by hyperglycemia, in pancreatic islets. These results suggest that SGLT2 inhibitors improve the sensitivity of beta cells to incretins.

Amelioration of glucose toxicity is one possible mechanism that may underlie the beneficial effects of SGLT2 inhibitors on beta cells. Given that such drugs attenuate insulin resistance [14], the resulting relief from the demand for excessive insulin production, a well-known trigger of endoplasmic reticulum stress and oxidative stress in pancreatic beta cells [15], is also likely related to their effects on beta cell function. In addition, lipotoxicity, which also impairs beta cell function [16], might be ameliorated by SGLT2 inhibitors via an improvement in systemic lipid metabolism [17]. Given that the entire repertoire of humoral and metabolic effects of SGLT2 inhibitors has likely not yet been recognized, additional mechanisms also may contribute to their protective actions. In this regard, SGLT2 inhibitors increase the production of ketone bodies, which may improve energy metabolism in cardiac muscle cells and thereby augment cardiac function [18]. Furthermore, whereas the underlying mechanism remains obscure, SGLT2 inhibitors increase the hematocrit in a manner independent of a decrease in circulating plasma volume [19]. This action also may contribute to the protective effects of these drugs on cardiac and renal function [19]. Further investigation of the actions of SGLT2 inhibitors is thus warranted to provide a more complete understanding of their antidiabetic and many other clinically beneficial effects.

\section{Compliance with ethical standards}

Conflict of interest WO has received lecture fees and research support from Astellas, AstraZeneca, Boehringer Ingelheim, Daiichi Sankyo, Mitsubishi Tanabe Pharma, MSD, Novartis, and Sanofi.

Human rights statement and informed consent This article does not contain any original studies with human or animal subjects performed by any of the authors.

\section{References}

1. Butler AE, Janson J, Bonner-Weir S, et al. Beta-cell deficit and increased beta-cell apoptosis in humans with type 2 diabetes. Diabetes. 2003;52:102-10.
2. Saisho Y, Butler AE, Manesso E, et al. $\beta$-cell mass and turnover in humans: effects of obesity and aging. Diabetes Care. 2013;36:111-7.

3. Kou K, Saisho Y, Satoh S, et al. Change in $\beta$-cell mass in Japanese nondiabetic obese individuals. J Clin Endocrinol Metab. 2013;98:3724-30.

4. Zinman B, Wanner C, Lachin JM, et al. Empagliflozin, cardiovascular outcomes, and mortality in type 2 diabetes. $\mathrm{N}$ Engl J Med. 2015;373:2117-28.

5. Neal B, Perkovic V, Mahaffey KW, et al. Canagliflozin and cardiovascular and renal events in type 2 diabetes. N Engl J Med. 2017;377:644-57.

6. Merovci A, Mari A, Solis C, et al. Dapagliflozin lowers plasma glucose concentration and improves $\beta$-cell function. J Clin Endocrinol Metab. 2015;100:1927-32.

7. Al Jobori H, Daniele G, Adams J, et al. Empagliflozin treatment is associated with improved $\beta$-cell function in type 2 diabetes mellitus. J Clin Endocrinol Metab. 2018;103:1402-7.

8. Takahara M, Shiraiwa T, Matsuoka T, et al. Ameliorated pancreatic $\beta$ cell dysfunction in type 2 diabetic patients treated with a sodium-glucose cotransporter 2 inhibitor ipragliflozin. Endocrine J. 2015;62:77-86.

9. Hansen HH, Jelsing J, Hansen CF, et al. The sodium glucose cotransporter type 2 inhibitor empagliflozin preserves $\beta$-cell mass and restores glucose homeostasis in the male Zucker diabetic fatty rat. J Pharmacol Exp Ther. 2014;350:657-64.

10. Okauchi S, Shimoda M, Obata A, et al. Protective effects of SGLT2 inhibitor luseogliflozin on pancreatic $\beta$-cells in obese type 2 diabetic db/db mice. Biochem Biophys Res Commun. 2016;470:772-82.

11. Cheng ST, Chen L, Li SY, et al. The effects of empagliflozin, an SGLT2 inhibitor, on pancreatic $\beta$-cell mass and glucose homeostasis in type 1 diabetes. PLoS ONE. 2016;11:e0147391.

12. Ahn $\mathrm{CH}$, Oh TJ, Kwak SH, et al. Sodium-glucose cotransporter-2 inhibition improves incretin sensitivity of pancreatic $\beta$-cells in people with type 2 diabetes. Diabetes Obes Metab. 2018;20:370-7.

13. Xu G, Kaneto H, Leybutt DR, et al. Downregulation of GLP-1 and GIP receptor expression by hyperglycemia. Diabetes. 2007;56:1551-8.

14. Xu L, Nagata N, Nagashimada M, et al. SGLT2 inhibition by empagliflozin promotes fat utilization and browning and attenuates inflammation and insulin resistance by polarizing M2 macrophages in diet-induced obese mice. EbioMedicine. 2017;20:137-49.

15. Szabat M, Page MM, Panzhinskiy E, et al. Reduced insulin production relieves endoplasmic reticulum stress and induces $\beta$ cell proliferation. Cell Metab. 2016;23:179-93.

16. Lalloyer F, Vandewalle B, Percevault F, et al. Peroxisome proliferator-activated receptor alpha improves pancreatic adaptation to insulin resistance in obese mice and reduces lipotoxicity in human islets. Diabetes. 2006;55:1605-13.

17. Hayashi T, Fukui T, Nakanishi N, et al. Dapagliflozin decreases small dense low-density lipoprotein-cholesterol and increases high-density lipoprotein 2-cholesterol in patients with type 2 diabetes: comparison with sitagliptin. Cardiovasc Diabetol. 2017; $16: 8$

18. Ferrannini E, Mark M, Mayoux E. CV protection in the EMPAREG OUTCOME Trial: a "thrifty substrate" hypothesis. Diabetes Care. 2016;39:1108-14.

19. Sano M, Takei M, Shiraishi Y, Suzuki Y. Increased hematocrit during sodium-glucose cotransporter 2 inhibitor therapy indicates recovery of tubulointerstitial function in diabetic kidneys. J Clin Med Res. 2016;8:844-7. 